\title{
Genetics of eye diseases
}

\section{Preface}

This issue features a wide array of articles relating to genetic as well as molecular and cellular aspects of eye diseases. The eye itself is a microcosm in terms of structure organization and function and hence, the theme of 'eye disease' is necessarily very diverse. It includes diseases that affect a wide range of tissues that together perform the function of vision-from the highly specialized, multiple types of neurons that constitute the retina which is the light sensitive layer, the pigmented cells of the choroid, the supporting vasculature, to the transparent refractive structures of lens and cornea-varied yet complementary in their roles. The field of ophthalmic genetics encompasses this diversity not only in the location and manifestation of different eye diseases but also in the spectrum of genetic causation. While the field has been predominantly concerned with rare Mendelian or single gene disorders in the past, the current decade has seen a burgeoning of the genetics of the more common complex eye diseases. This trend reflects that of human genetics as a whole. Technological advances over the late 20th century have made it feasible to dissect genetic components of varying magnitudes that make up the etiology of complex diseases. Questions relating to the role of genes in the etiology of common complex diseases are as yet largely unanswered and the current challenges facing ophthalmic genetics are in understanding of the nature of genetic variations as well as their role in causation of complex eye diseases such as adult-onset glaucoma, age-related macular degeneration and age-related cataract. On the other hand, the tools that have been established and currently available for studying Mendelian disorders have resulted in the identification of a large number of genes underlying various forms of the inherited ocular disorders such as corneal dystrophy, congenital cataract, retinal degeneration and congenital and juvenile glaucoma. The rapid growth in identification of genes in this group of eye diseases is illustrated very clearly in the case of retinal degenerations, which are a large group of very heterogeneous diseases of the retina, for which the number of mapped and/or identified genes grew from none to almost 200 in spanning most of the last three decades.

What are the current and future directions for the genetics of eye disorders and what are its aims beyond just identification of genes? It would not be easy to provide a common answer to the vast array of ocular diseases; rather one needs to explore the relevant areas within the framework of each specific disease. There are obvious lacunae in the knowledge of genes underlying monogenic eye disorders that are yet to be filled. For example, genetic heterogeneity in Mendelian disorders is a significant obstacle to identification of all genes responsible for diseases such as retinitis pigmentosa (RP). Despite identification of $>40$ genes for non-syndromic RP, these do not account for disease in a large proportion of patients. Other complexity of the simple monogenic diseases stems from our inability to establish one-to-one correlation of genotype of the patient with his pathogenic phenotype. The basis for phenotypic variability between individuals having the same gene mutation is not well-understood at present and thought to be influenced by interplay of other genetic (e.g. modifier locus) and environmental factors. While the application of genetics for Mendelian disorders is straightforward in principle for diagnostic screening, there are technical challenges in achieving this in a cost-effective and efficient manner. For example, genetic heterogeneity of RP poses a challenge to genetic testing of patients for mutations. Similar considerations would apply to gene-specific approaches to gene therapy as well.

Since the goal of all gene identification endeavors is ultimately to understand the pathogenesis of the disease, a major task accompanying gene discovery is functional genomics. This involves investigation of functions and interactions of the proteins involved and the consequences of mutation at the biochemical and sub-cellular levels. This is a continuous effort as more genes are identified for ocular diseases.

In this issue, the theme of genetics of eye diseases is covered by articles that review the genetics of several disorders and also highlight areas of current interest in molecular pathogenesis and therapy in relation to eye diseases.

Elias Traboulsi (page 395) provides a brief perspective on the potential of advances in ophthalmic genetics to aid in clinical management and therapy of ocular diseases with special reference to retinal degenerations or dystrophies.

Looking at the roles of different photoreceptor ciliary proteins in photoreceptor function as well as in retinal dystrophy phenotypes, Hemant Khanna and co-workers (page 399) review current status of the genetics and protein interactions involving retinitis pigmentosa GTPase-regulator (RPGR) and its 'interactome'. 
Therapeutic approaches for retinal degenerations being actively explored include gene therapy as well as cell-based therapies that utilize stem cells or pluripotent cells for replacement of damaged photoreceptors. Hiroshi Tomita et al. (page 409) provide an overview of a specific type of molecule with phototransducing properties (the channel-rhodopsins), and their potential in gene therapy. The article by Masayo Takahashi and co-workers (page 417) looks at the emerging technology of induced pluripotent cells (iPS) and its scope as a model system to understand the mechanisms of retinal degenerative diseases as well as a therapeutic avenue.

Two important and challenging areas in the genetics of complex eye diseases are covered in two comprehensive reviews in this issue. In an article on age-related macular degeneration (AMD), Subhabrata Chakrabarti and co-workers (page 425) have provided an overview on the underlying molecular genetic aspects of the disease and highlighted AMD-associated candidate genes and their potential role in disease pathogenesis. A review of the genetics of primary open angle glaucoma (POAG) by Kunal Ray and coworkers (page 451) examines current understanding of the genetics of POAG and attempts to provide a holistic view on the potential of multiple biochemical pathways that appears to be influencing the disease pathogenesis based on the information available in the current literature.

Animal models provide valuable insights into genetics and pathogenic mechanisms of eye diseases. The review of mouse models for cataract by Jochen Graw (page 469) gives a detailed account of several mouse models of cataract and the genes involved, highlighting the role of these genes in human hereditary cataracts as well.

Hereditary corneal disorders are a heterogeneous group of disorders consisting of phenotypes with both Mendelian and complex inheritance. The article by Chitra Kannabiran (page 487) reviews the current status of genetics of corneal dystrophies affecting the corneal endothelium.

Regulation of angiogenesis is a process that has important implications in the pathogenesis of several eye diseases. The article by Yureeda Qazi and co-workers (page 495) deals with molecules and pathways involved in angiogenesis and their roles in different disorders affecting the cornea and retina.

Ultimately, our knowledge needs to translate to clinical benefits for patients either through robust genetic screening to detect the causative genes in the population in case of ocular diseases or through surgical and/or therapeutic intervention as appropriate. In addition, the advancement of gene and stem cell therapies are likely to break new grounds in the way the eye diseases will be combated and controlled. Understanding the underlying molecular events in the pathogenesis of eye diseases could provide key leads to prevention. We hope that the current trends in ophthalmic genetics would pave the way to this larger goal.

E-mail: chitra@lvpei.org; chitra_kannabiran@rediffmail.com; kunalray@gmail.com

Chitra Kannabiran

KUNAL RAY

Editors 